\title{
Molecular Cloning, Sequence Analysis and Expression Analysis of an NtWRKY6 from Tobacco (Nicotiana tabacum L.) in Abiotic Strsss
}

\author{
Liqin Li, Yubi Huang*, Wei Wang, Yaling Xu \\ College of Agronomy, Sichuan Agricultural University, Chengdu, China. \\ Email: *yubihuang@sohu.com
}

Received August 14 $4^{\text {th }}, 2012$; revised September $17^{\text {th }}, 2012$; accepted October $8^{\text {th }}, 2012$

\begin{abstract}
In higher plants, WRKY gene family plays a significant role in transcriptional regulation. They are widely involved in biotic stress, abiotic stress, growth, development and metabolism regulation. In this study WRKY6 from tobacco is cloned by homology cloning. 1647 nucleotide sequences were obtained. The deduced protein sequences show that this protein belongs to the second group of WRKY family, only have one WRKY structure, and the zinc-finger structure is $\mathrm{C}-\mathrm{X}_{4}-\mathrm{C}-\mathrm{X}_{23}-\mathrm{H}-\mathrm{X}_{1}-\mathrm{H}$. Phylogeny results show NtWRKY6 is much closer to NtWRKY1 generated $97 \%$ amino acids similarity. RT-PCR analysis has revealed that expression levels of NtWRKY6 has increased rapidly at $3 \mathrm{~h}$ under NaCI and PEG treatment. The results suggest that NtWRKY6 is an early responder and may be involved in $\mathrm{NaCl}$ and PEG abiotic stress in tobacco.
\end{abstract}

Keywords: WRKY Protein; Molecular Cloning; Tobacco; RT-PCR; Expression Analysis

\section{Introduction}

WRKY protein was originally found in sweet potato and named SPF1 [1], so far, this family protein was also found in many plants such as Arabidopsis thaliana, potato, Gossypium spp, Oryza sativa; Nicotiana tabacum [2-6]. Until the 2005 year, WRKY homologues from two non-plant eukaryotic species Giardia lamblia and Dictyostelium discoideum were found [7]. WRKY proteins have been reported to be involved in defense against bacteria, fungi $[8,9]$, wounding [10], senescence [11], development [12] and abiotic stresses [13,14].

WRKY family proteins are characterized by one or two highly conserved WRKYGQK domains, The WRKY domain is about 60 amino acids in length and a Cys(2) His(2) or Cys(2)HisCys zinc-binding motif [15]. The two motifs of WRKY proteins are necessary for the high binding affinity of WRKY proteins to DNA sequence motif (W-boxes), which contain an invariant DNA sequence $(\mathrm{T})(\mathrm{T}) \mathrm{TGAC}(\mathrm{C} / \mathrm{T})[16]$.

Abiotic stress is an important factor which affects plant growth, distribution, yield and quality in nature. NtWRKY6 was identified in the native tobacco Nicotiana attenuate, whose wound responses was significantly amplified when fatty acid amino acid conjugates

${ }^{*}$ Corresponding author.
(FACs) in larval oral secretions was introduced into wounds during feeding [17]. The report of NtWRKY6 involved in abiotic stresses had not been seen.

The tobacco Varieties K326 is the main cultivated species in the world, our researches first reported that WRKY6 gene was cloned from the species, 1647 nucleotide sequences were obtained. The deduced protein sequence showed that this protein belonged to the second group of WRKY family, only had one WRKY structure, and the zinc-finger structure was $\mathrm{C}-\mathrm{X}_{4}-\mathrm{C}-\mathrm{X}_{23}-\mathrm{H}-\mathrm{X}_{1}-\mathrm{H}$. RT-PCR analysis had revealed that expression levels of NtWRKY6 had increased rapidly at $3 \mathrm{~h}$ under $\mathrm{NaCI}$ and PEG abiotic stress treatment.

\section{Materials and Methods}

\subsection{Plant Materials and Sample Preparation}

Tobacco (Nicotiana tabacum L.) K326 was used in this study. Seeding was sown on MS medium under a $16-\mathrm{h}$ light $/ 8$-h dark cycle at $28^{\circ} \mathrm{C}$. When the second leaves were fully expanded, the tobacco tissues were collected for investigation or frozen in liquid nitrogen immediately, stored at $-80^{\circ} \mathrm{C}$ for further analysis. For NaCI and PEG treatment, the seeding when had second leaves were transferred to MS medium with $250 \mathrm{mM} \mathrm{NaCI}$ or $300 \mathrm{~m}$ M PEG for 0, 3, 6, 12, $24 \mathrm{~h}$ treatment. 


\subsection{Isolation of the Tobacco WRKY6 Gene}

The specific primers of NtWRKY6 were designed according to the published sequences (Genebank accession number: AY456272.1). The sequences of primers were shown in Table 1. PCR product was purified and subcloned into PMD-19T vector (Takara) for sequencing.

\subsection{Bioinformatics Analysis}

Sequence analysis was carried out by Blast software (http://www.ncbi.nlm.gov/blast). Deduced anino acid sequences were aligned using Clustal W (http://npsa-pbil. ibcp.fr) software [18]. A phylogenetic tree was constructed based on full-length amino acid sequences using the neighbor-joining method [19] and was drawn using MEGA Version 4.1 [20].

\subsection{RNA Extraction and Reverse Transcription RT-PCR}

Total RNA was extract from fresh or frozen samples of tobacco using Trizol reagent (Invitrogen) according to the standard protocol. In addition, total RNA was also extracted from tobacco seeding at $0,3,6,12,24 \mathrm{~h}$ after $\mathrm{NaCI}$ or PEG treatment. First-strand cDNA was synthesized with the extracted RNA using M-MLV reverse transcriptase (Promega) at $42^{\circ} \mathrm{C}$ for $1 \mathrm{~h}$ and used as the template for PCR [21]. Amplification was performed using the following cycling parameters: $95^{\circ} \mathrm{C}$ for $5 \mathrm{~min}$ followed by 35 cycles of $95^{\circ} \mathrm{C}$ for $30 \mathrm{~S}$ (denaturation), $55^{\circ} \mathrm{C}$ for $30 \mathrm{~S}$ (annealing), $72^{\circ} \mathrm{C}$ for $1 \mathrm{~min}$ and $40 \mathrm{~S}$ (elongation). After the reaction, PCR products were analyzed on $1.2 \%(\mathrm{w} / \mathrm{v})$ agrose gels. Expression levels were assessed using $18 \mathrm{~S}$ rDNA as internal standards for normalization of sample variation. The primers of NtWRKY6 and 18SrDNA for RT-PCR analysis are shown in Table 1.

\section{Results}

\subsection{Amplification of Full Length cDNA of NtWRKY6}

To clone the full length cDNA of NtWRKY6. The full

Table 1. Primer sequences and their uses.

\begin{tabular}{|c|c|c|}
\hline Primer name & Nucleotide sequence $\left(5^{\prime} \rightarrow 3^{\prime}\right)$ & Purpose \\
\hline NtWRKY6-F1 & ATGAATTCTTTTACTAGCAA & \\
\hline NtWRKY6-R1 & TCAGTTAAGGAAAGAGCTGA & amplific \\
\hline NtWRKY6-F2 & TTCTTTTGGTGACGAGGACA & \multirow{2}{*}{$\begin{array}{l}\text { RT-PCR } \\
\text { expression } \\
\text { analysis }\end{array}$} \\
\hline NtWRKY6-R2 & CAAATCCTGA ATAACTTGAA & \\
\hline 18SrDNA-F & CCTACGCTCTGTATACATTA & \multirow{2}{*}{$\begin{array}{l}\text { RT-PCR } \\
\text { expression } \\
\text { analysis }\end{array}$} \\
\hline 18SrDNA-R & GTGTTGAGTCAAATTAAGCC & \\
\hline
\end{tabular}

length primers of NtWRKY6 were used to amplify this gene, RT-PCR products were analyzed on $1.2 \%(\mathrm{w} / \mathrm{v})$ agarose gels, and obtained the expected size fragment (Figure 1). PCR product was purified and subcloned into PMD-19T vector (Takara) for sequencing using three positive clones. The result of sequencing had revealed the amplification product of PCR had 1647 base pairs (bp).

\subsection{Homologous Alignment and Phylogenetic Analysis of NtWRKY6}

To better understand the structure of the deduced amino acid sequence encoded by tobacco WRKY6, a homologous alignment was carried out with Clustal $\mathrm{W}$ program using the amino acid sequences of WRKY 6 and 19 proteins (Figure 2). Sequence alignment revealed that all proteins discussed above appeared to be rather conserved in structure. These proteins all had only one WRKY structure (WRKYGQK) and the zinc-finger structure is C-X4-C-X23-H-X1-H. Additionally, the NtWRKY6 shared a very high identity of NtWRKY1 (BAA82107.1) with $97 \%$, and lower identity of ZmWRKY53 (NP 001147949.1) and Gm WRKY2 (XP 003526383.1) with $41 \%$.

In order to determine the relationship between WRKY6 and 19 proteins, phylogenetic analysis was further carried out by Clustal W program with default parameters using the amino acid sequences. The phylogenetic relationship among 20 WRKY Protein (Figure 3) showed that the NtWRKY6, NtWRKY1, PcWRKY1 and HaWRKY7 had relatively close relationship. Fourthermore, the NtWRKY6, ZmWRKY53 and OsWRKY53 had a relatively far relationship.

\subsection{Expression Levels of NtWRKY6 in Different Tissues}

In order to get an insight into the potential function of NtWRKY6 in tobacco, tissue distribution of NtWRKY6

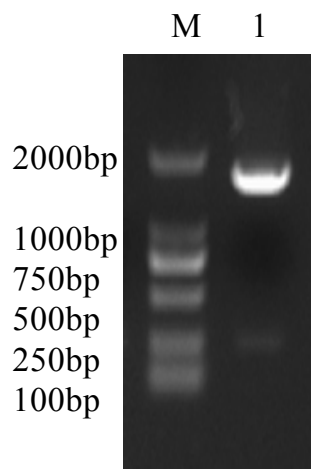

Figure 1. RT-PCR result of NtWRKY6. M DL2000 DNA molecular marker, 1 product of RT-PCR. 


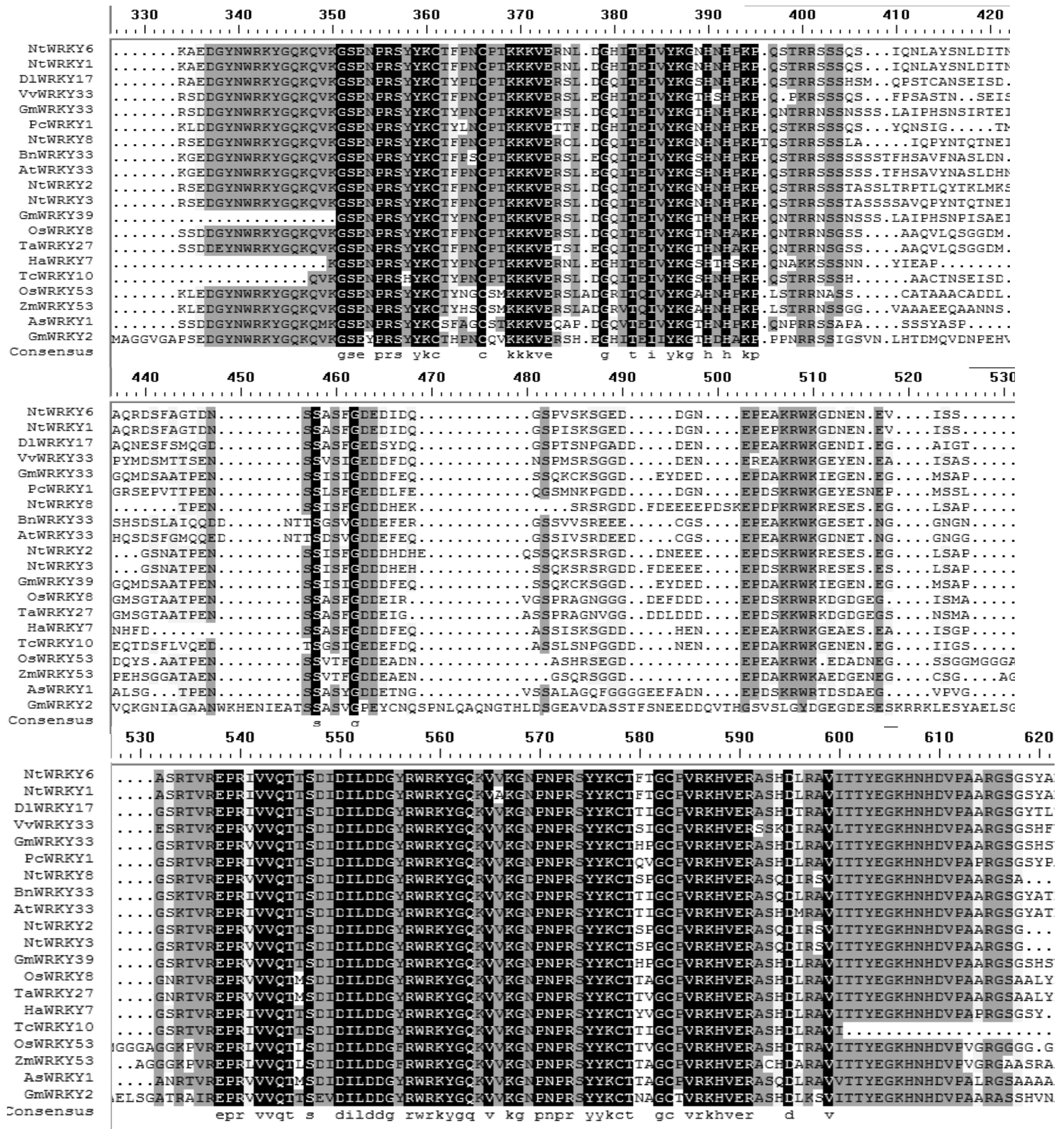

Figure 2. An Alignments of the NtWRKY6 conserved amino acid sequences with homologous 19 WRKY proteins. NtWRKY1 (GenBank accession no, BAA82107.1), DIWRKY17 (GenBank accession no, AEO31478.1), VvWRKY33 (GenBank accession no, XP_002264974.1), GmWRKY33 (GenBank accession no, XP_003544908.1), PcWRKY1 (GenBank accession no, AAC49527.1), NtWRKY7 (GenBank accession no, AEX49954.1), BnWRKY33 (GenBank accession no, ACI14397.1), AtWRKY33 (GenBank accession no, AAM34736.1), NtWRKY2 (GenBank accession no, BAA77383.1), NtWRKY3 (GenBank accession no, AAS13439.1), GmWRKY39 (GenBank accession no, ABS18436.1), OsWRKY8 (GenBank accession no, BAB61266.1), TaWRKY27 (GenBank accession no, ACD80363.1), HaWRKY7 (GenBank accession no, AFL91071.1), TaWRKY10 (GenBank accession no, AAQ57645.1), OsWRKY53 (GenBank accession no, DAA05118.1), ZmWRKY53 (GenBank accession no, NP_001147949.1), AsWRKY1 (GenBank accession no, AAD32677.1), GmWRKY2 (GenBank accession no, XP_003526383.1), were aligned by Clustal program version 1.83. According to the degree of conservation, dark gray and light gray are indicated. 


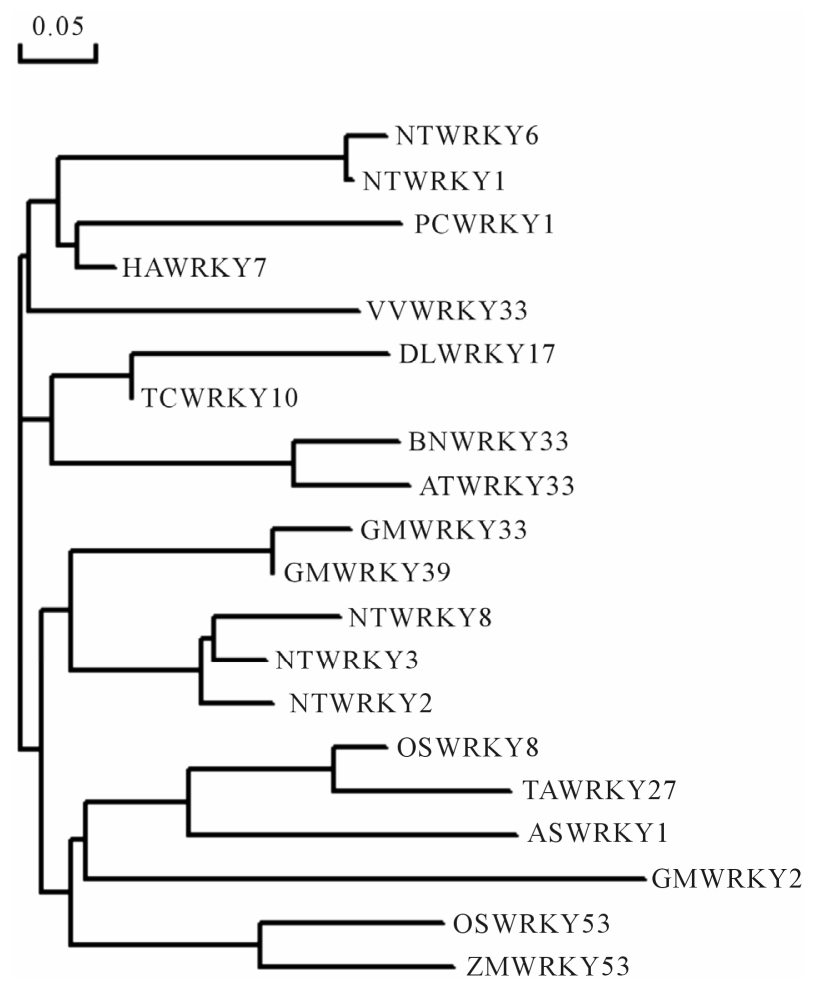

Figure 3. Phylogenetic analysis of the deduced amino acid sequence between NtWRKY6 and 19 WRKY protein.

was studied by semi-quantitative RT-PCR (Figure 4). The results showed that NtWRKY6 was ubiquitously expressed in tobacco tissues including root, stem, leaf, and flower. NtWRKY6 transcripts seemed to be much abundant in root, leaf, and flower. At the same time, the lower expression level in stem.

\subsection{Stress Induced Expression Patterns of NtWRKY6}

To further investigate whether the expression of NtWRKY6 was induced by abiotic stresses, we first monitored the mRNA transcript level of NtWRKY6 under different abiotic stress treatments, including $\mathrm{NaCl}$ and PEG by RT-PCR (Figures 5 and 6). As shown in Figure 5, the expression level of NtWRKY6 transcripts under $\mathrm{NaCl}$ stress reached the highest level at $3 \mathrm{~h}$. It kept at 6,12 h and decreased at $24 \mathrm{~h}$. As shown in Figure 6, the expression level of NtWRKY6 transcripts under PEG stress reached the highest level at $3 \mathrm{~h}$. Then, decreased gradually at $6,12 \mathrm{~h}$, and declined rapidly at $24 \mathrm{~h}$. These results revealed the expression level of the NtWRKY6 was increased rapidly at $3 \mathrm{~h}$ by the $\mathrm{NaCl}$ and PEG stress treatment, suggesting NtWRKY6 was an early responders and may be involved in $\mathrm{NaCl}$ and PEG stress in tobacco. Clearly, this will be an important clue for future investigation.

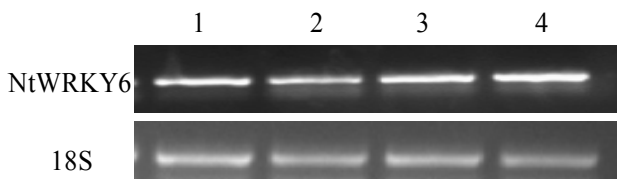

Figure 4. RT-PCR analysis of expression level of NtWRKY6 in tobacco tissues using the specific primers of NtWRKY6. $18 \mathrm{~S}$ was used for endogenous control. 1: root; 2: stem; 3 : leaf; 4: flower.

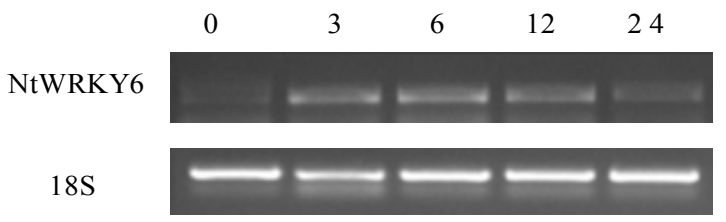

Figure 5. RT-PCR analysis of expression level of NtWRKY6 in tobacco seeding at $0,3,6,12$ and $24 \mathrm{~h}$ after $250 \mathrm{mM}$ NaCI treatment. $18 \mathrm{~S}$ was used for endogenous control.

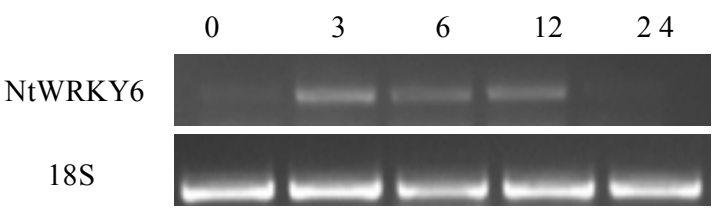

Figure 6. RT-PCR analysis of expression level of NtWRKY6 in tobacco seeding at $0,3,6,12$ and $24 \mathrm{~h}$ after $300 \mathrm{mM}$ PEG treatment. $18 \mathrm{~S}$ was used for endogenous control.

\section{Discussion}

According to WRKY domain number and zinc-binding motif type, WRKY protein were divided three groups, group I members contained two WRKY domains and contained a Cys2His2 zinc-finger motif. Group II and group III contained one WRKY domain, the second group zinc-binding motif type was same to the first, group III zinc-binding motif was Cys2HisCys zinc-finger motif [15]. Its amino acids sequence was different from group I and group II. At the present, most of WRKY proteins were the number of the group II, it was speculated that the first group WRKY proteins were produced because of WRKY domain lost and mutation, the third group was produced, probably $\mathrm{H}$ was mutated to $\mathrm{C}$ in zinc-binding motif type of the second group. So group I was the most conservative and group III was the most active in evolutionary history of WRKY family [22]. Previous studies about some WRKY proteins of tobacco had indicated their roles in biotic stress. For instance, the expression level of NtWIZZ (Wound-induced Leucine Zipper Zinc finger) was rapid accumulation at ten minutes and arrived peak at $30 \mathrm{~min}$ upon wounding [10]. NtWRKY1 and NtWRKY2 had the same expression pattern with NtWIZZ upon wounding [23].

NtTIZZ was an early responsive gene and hypersensitive responders to tobacco mosaic virus, this protein 
maybe play an important role in defense response [24]. NtWRKY3 and NtWRKY9 were induced by pathogen and salicylic acid, this suggested they were participated in pathogen and salicylic acid pathway [6]. NtWRKY3 was downstream genes of MAPK, silencing of tobacco WRKY8 decreased the expression of defense-related genes and increased disease susceptibility to the pathogens Phytophthora infestans and Colletotrichum orbiculare [25]. Only one report about WRKY from tobacco was involved in abiotic stress, which had showed a WRKY gene expression was active by drought stress and heat shock together, but not by only one factors [26].

We had cloned NtWRKY6 from tobacco Varieties K326, NtWRKY6 encoded a protein of 549 amino acids. Phylogenetic analysis showed that the NtWRKY6, NtWRKY1, PcWRKY1 and HaWRKY7 had a relatively close relationship. RT-PCR analysis had revealed that NtWRKY6 was ubiquitously expressed in tobacco tissues including root, stem, leaf, and flower. To further explore the function of NtWRKY6, RT-PCR was also used to examine the expression of NtWRKY6 under $\mathrm{NaCl}$ and PEG treatments for $0,3,6,12,24 \mathrm{~h}$. we had observed that the expression of NtWRKY6 reached the highest level at $3 \mathrm{~h}$. It kept $6,12 \mathrm{~h}$ and decreased at $24 \mathrm{~h}$ under $\mathrm{NaCl}$ treatment, indicating that NtWRKY6 was a rapidly responder to $\mathrm{NaCl}$ stress. Previous studies had detected that transcript levels of BcWRKY46 (Brassica campestrisssp Chinensis.) was induced after $2 \mathrm{~h}$ of 150 $\mathrm{mM} \mathrm{NaCl}$ treatment with real-time RT-PCR [27].

Drought is one of the major factors to limit the plant growth and development in China, the expression level of NtWRKY6 transcripts under PEG stress reached the highest level at $3 \mathrm{~h}$. Then, decreased gradually at $6,12 \mathrm{~h}$, and declined rapidly at $24 \mathrm{~h}$. This result showed that NtWRKY6 was participated in drought stress. Recently there are many researches about WRKY genes involved in PEG stress, such as Zhang have showed the transcription of HbWRKY1 (Hevea brasiliensis Muell. Arg.) in leaves was gradually up-regulated and came to its peak at $12 \mathrm{~h}$ after 20\% PEG 6000 treatment [28]. So, we can conclude NtWRKY6 might be upstream regulator in salt and drought pathway. When tobacco faces this two stress, the expression level of NtWRKY6 rising will increase or decrease downstream target gene expression, some researches had reported the expression of BcWRKY46 (Brassica campestrisssp Chinensis.) and LtWRKY21 (Larrea tridentata) were both enhanced by drought and high salinity [27,29]. On the other hand, under drought and salt stress, no obviously change of the GhWRKY3 expression was observed [30]. OsWRKY80 expression was up-regulated by drought stress and there was no change under $100 \mathrm{mM} \mathrm{NaCl}$ stress [31]. These results suggested WRKY protein from different plants had many different expression patterns and functions.

In conclusion, we have isolated and characterized NtWRKY6 from tobacco (Nicotiana tabacum L.), then have examined the tissue distribution and expression pattern of NtWRKY6 with RT-PCR. According to our results, $\mathrm{NtWRKY6}$ may play a vital role in the $\mathrm{NaCl}$ and PEG stress of tobacco.

However, for further study, detailed investigations should be carried out to clarify the function of NtWRKY6, such as acquiring transgenic plants of over-expression NtWRKY6 and prokaryotic expression NtWRKY6, these experiments will be very important in our future work.

\section{Acknowledgements}

This work was supported by Natural Science Foundation of China (No. 31070244).

\section{REFERENCES}

[1] S. Ishiguro and K. Nakamura, "Characterization of a cDNA Encoding a Novel DNA-Binding Protein, SPF1, That Recognizes 1SP8 Sequences in the 50 Upstream Regions of Genes Coding for Sporamin and Beta-Amylase from Sweet Potato," Molecular Genetics and Genomics, Vol. 244, No. 6, 1994, pp. 563-571. doi:10.1007/BF00282746

[2] S. Robatzek and I. E. Somssich, "A New Member of the Arabidopsis WRKY Transcription Factor Family, AtWRKY6, Is Associated with Both Senescence- and Defense-Related Processes," The Plant Journal, Vol. 28, 2001, pp. 123-133.

[3] M. Lagacé and D. P. Matton, "Characterization of a WRKY Transcription Factor Expressed in Late Torpedo Stage Embryos of Solanum chacoense," Planta, Vol. 219, No. 1, 2004, pp. 185-189. doi:10.1007/s00425-004-1253-2

[4] Y. H. Xu, J. W. Wang, S. Wang, J. Y. Wang and X. Y. Chen, "Characterization of GaWRKY1, a Cotton Transcription Factor That Regulates the Sesquiterpene Synthase Gene $(+)-\delta$-Cadinene Synthase-A," Plant Physiology, Vol. 135, No. 1, 2004, pp. 507-515. doi:10.1104/pp.104.038612

[5] Z. L. Zhang, Z. Xie, X. L. Zou, J. Casaretto, T. H. David and Q. X. Shen, "A Rice WRKY Gene Encodes a Transcriptional Repressor of the Gibberellin Signaling Pathway in Aleurone Cells," Plant Physiology, Vol. 134, No. 4, 2004, pp. 1500-1513.

[6] C. Chen and Z. Chen, "Isolation and Characterization of Two Pathogen and Salicylic Acid-Induced Genes Encoding WRKY DNA-Binding Proteins from Tobacco," Plant Molecular Biology, Vol. 42, No. 2, 2000, pp. 387-396. doi:10.1023/A:1006399311615

[7] Y. Zhang and L. Wang, "The WRKY Transcription Factor Superfamily: Its Origin in Eukaryotes and Expansion in Plants," BMC Evolutionary Biology, Vol. 5, 2005, pp. 1-12. doi:10.1186/1471-2148-5-1 
[8] P. Z. Yang, C. H. Chen, Z. P. Wang, B. F. Fan and Z. X. Chen, "A Pathogen and Salicylic Acid-Induced WRKY DNA-Binding Activity Recognizes the Elicitor Response Element of the Tobacco Class I Chitinase Gene Promoter," The Plant Journal, Vol. 18, No. 2, 1999, pp. 141149.

[9] L. Du and Z. Chen, "Identification of Genes Encoding Receptorlikeprotein Kinases as Possible Targets of Pathogen-Andsalicylic Acid-Induced WRKY DNA-Binding Proteins in Arabidopsis," The Plant Journal, Vol. 24, No. 6, 2000, pp. 837-847.

[10] K. Hara, M. Yagi, T. Kusano and H. Sano, "Rapid Systemic Accumulation of Transcripts Encoding a Tobacco WRKY Transcription Factor upon Wounding," Molecular Genetics and Genomics, Vol. 263, No. 1, 2000, pp. 30-37. doi:10.1007/PL00008673

[11] S. Robatzek and I. E. Somssich, "Targets of AtWRKY6 Regulation during Plant Senescence and Pathogen Defense," Genes \& Development, Vol. 16, 2002, pp. 11391149. doi:10.1101/gad.222702

[12] M. Luo, E. S. Dennis, F. Berger, W. J. Peacock and A. Chaudhury, "A MINI SEED3 (MINI3), a WRKY Family Gene, and HAIKU2 (IKU2), a Leucine-Rich Repeat (LRR) Kinase Gene, Are Regulators of Seed Size in Arabidopsis," Proceedings of the National Academy of Sciences of the USA, Vol. 102, No. 48, 2005, pp. 1753117536. doi:10.1073/pnas.0508418102

[13] B. N. Devaiah, A. S. Karthikeyan and K. G. Raghothama, "WRKY75 Transcription Factor Is a Modulator of Phosphate Acquisition and Root Development in Arabidopsis," Plant Physiology, Vol. 143, No. 4, 2007, pp. 17891801.

[14] Y. F. Chen, L. Q. Li, Q. Xu, Y. H. Kong, H. Wang and W. $\mathrm{H}$. Wu, "The WRKY6 Transcription Factor Modulates PHOSPHATE1 Expression in Response to Low Pi Stress in Arabidopsis," The Plant Cell, Vol. 21, No. 11, 2009, pp. 3554-3566. doi:10.1105/tpc.108.064980

[15] T. Eulgem, P. J. Rushton, S. Robatzek and I. E. Somssich, "The WRKY Superfamily of Plant Transcription Factors," Trends in Plant Science, Vol. 5, No. 5, 2000, pp. 199-206. doi:10.1016/S1360-1385(00)01600-9

[16] P. J. Rushton, H. Macdonald, A. K. Huttly, C. M. Lazarus and R. Hooley, "Members of a New Family of DNABinding Proteins Bind to a Conserved cis-Element in the Promoters of Alpha-Amy2 Genes," Plant Molecular Biology, Vol. 29, No. 4, 1995, pp. 691-702.

[17] M. Skibbe, N. Qu, I. Galis and I. T. Baldwin, "Induced Plant Defenses in the Natural Environment: Nicotiana Attenuata WRKY3 and WRKY6 Coordinate Responses to Herbivory," The Plant Cell, Vol. 20, No. 7, 2008, pp. 1984-2000. doi:10.1105/tpc.108.058594

[18] Y. L. Zhuang, G. J. Ren, C. M. He, X. Y. Li, Q. M. Meng, C. F. Zhu, R. C. Wang and J. Zhang, "Cloning and Characterization of a Maize cDNA Encoding Glutamate Decarboxylase," Plant Molecular Biology Reporter, Vol. 28, No. 4, 2010, pp. 620-626.

[19] X. M. Liu, J. M. Anderson and P. M. Pijut, "Cloning and Characterization of Prunus serotina AGAMOUS, a Puta- tive Flower Homeotic Gene," Plant Molecular Biology Reporter, Vol. 28, No. 2, 2010, pp. 193-203. doi:10.1007/s11105-009-0140-1

[20] K. Tamura, J. Dudley, M. Nei and S. Kumar, "MEGA4: Molecular Evolutionary Genetics Analysis (MEGA) Software Version 4.0," Molecular Biology and Evolution, Vol. 24, No. 8, 2007, pp. 1596-1599.

[21] L. J. Wu, X. T. Wang, L. C. Wu, P. G. Wang and Y. H. Chen, "Molecular Cloning and Expression Analysis of an HINT1Homologue from Maize (Zea mays L.)," Plant Molecular Biology Reporter, Vol. 29, 2011, pp. 10061012.

[22] B. Ulker and I. E. Somssich, "WRKY Transcription Factors: From DNA Binding towards Biological Function," Current Opinion in Plant Biology, Vol. 263, No. 5, 2004, pp. 491-498. doi:10.1016/j.pbi.2004.07.012

[23] T. Nishiuchi, H. Shinshi and K. Suzuki, "Rapid and Transient Activation of Transcription of the ERF3 Gene by Wounding in Tobacco Leaves Possible Involvement of NtWRKYs and Auto Repression," Journal of Biological Chemistry, Vol. 279, No. 53, 2011, pp. 55355-55361. doi:10.1074/jbc.M409674200

[24] H. Yoda, M. Ogawa, Y. Yamaguchi, N. Koizumi, T. Kusano and H. Sano, "Identification of Early-Responsive Genes Associated with the Hypersensitive Response to Tobacco Mosaic Virus and Characterization of a WRKYType Transcription Factor in Tobacco Plants," Molecular Genetics and Genomics, Vol. 267, No. 2, 2002, pp. 154161. doi:10.1007/s00438-002-0651-z

[25] N. Ishihama, R. Yamada, M. Yoshioka, S. Katou and H. Yoshioka, "Phosphorylation of the Nicotiana Benthamiana WRKY8 Transcription Factor by MAPK Functions in the Defense Response," The Plant Cell, Vol. 23, No. 3, 2011, pp. 1153-1170.

[26] L. Rizhsky, H. Liang and R. Mittler, "The Combined Effect of Drought Stress and Heat Shock on Gene Expression in Tobacco," Plant Physiology, Vol. 130, No. 3, 2012, pp.1143-1151. doi:10.1104/pp.006858

[27] F. Wang, X. L. Hou, J. Tang, Z. Wang, S. M. Wang, F. Jiang and Y. Li, "A Novel Cold-Inducible Gene from Pak-Choi (Brassica campestrisssp. chinensis), BcWRKY46, Enhances the Cold, Salt and Dehydration Stress Tolerance in Transgenic Tobacco," Molecular Biology Reports, Vol. 39, No. 4, 2012, pp. 4553-4564. doi:10.1007/s11033-011-1245-9

[28] Q. Q. Zhang, J. H. Zhu, Y. M. Ni, Y. B. Cai and Z. L. Zhang, "Expression Profiling of HbWRKY1, an Ethephon-Induced WRKY Gene in Latex from Hevea brasiliensis in Responding to Wounding and Drought," Trees, Vol. 26, No. 2, 2012, pp. 587-595. doi:10.1007/s00468-011-0623-1

[29] X. L. Zou, Q. Shen and D. Neuman, “An ABA Inducible WRKYgene Integrates Responses of Creosote Bush (Larrea tridentata) to Elevated $\mathrm{CO}_{2}$ and Abiotic Stresses," Plant Science, Vol. 172, No. 5, 2004, pp. 997-1004. doi:10.1016/j.plantsci.2007.02.003

[30] R. Y. Guo, F. F. Yu, Z. Gao, H. L. An, X. C. Cao and X. Q. Guo, "GhWRKY3, a Novel Cotton (Gossypium hirsu- 
tum L.) WRKY Gene, Is Involved in Diverse Stress Responses," Molecular Biology Reports, Vol. 38, No. 1, 2011, pp. 49-58. doi:10.1007/s11033-010-0076-4

[31] K. Felipe, R. Ricachenevsky, S. Antonio, K. Paloma, J. Menguer and F. Palma, "Identification of Fe-Excess-In- duced Genes in Rice Shoots Reveals a WRKY Transcription Factor Responsive to Fe, Drought and Senescence," Molecular Biology Reports, Vol. 37, No. 8, 2010, pp. 3735-3745. 